\title{
Determination of nano-roughness for micro-objects by measuring the van der Waals force
}

\author{
Bratina, B..$^{a}{ }^{*}$, Šafarič, J. ${ }^{a}$, Uran, S. ${ }^{a}$, Šafarič, R. ${ }^{a}$ \\ ${ }^{\mathrm{a}}$ Faculty of Electrical Engineering and Computer Science, University of Maribor, Maribor, Slovenia
}

\begin{abstract}
A B S T R A C T
3D printing or assembly techniques in the micro/nano-world enable production of micro-parts for building small machines or structures for biomedicine applications, such as cultivation of living cells in the field of Tissue Engineering. Micro-sized assembly requires automated manipulation procedures and methods for determination of suitable objects for assembly. The latter is possible by van der Waals force measurement and determination of distance at the van der Waals peak between two objects in contact. They are dependent not only on the Hamaker coefficients of the materials in contact and their geometries, but also on the nano-roughness asperities and crystal structure asperities of the contact surfaces. A method is presented for measuring van der Waals' force and determining micro-objects' (sizes between 10-100 $\mu \mathrm{m}$ ) distances between materials in contact at the van der Waals peak in the presence of nano-roughness and crystal structure roughness. The proposed model was validated by experimental lab results between various materials and shapes (glass and polystyrene beads, metallic wires).
\end{abstract}

\section{ARTICLE INFO}

Keywords:

Micro-object;

Surface roughness;

Nano-roughness;

Van der Waals force;

Distance at van der Waals peak

*Corresponding author: bozidar.bratina@um.si (Bratina, B.)

Article history:

Received 14 September 2018

Revised 18 February 2019

Accepted 24 February 2019

\section{References}

[1] Ngo, T.D., Kashani, A., Imbalzano, G., Nguyen, K.T.Q., Hui, D. (2018). Additive manufacturing (3D printing): A review of materials, methods, applications and challenges, Composites Part B: Engineering, Vol. 143, 172-196, doi: 10.1016/j.compositesb.2018.02.012.

[2] Ru, C., Luo, J., Xie, S., Sun, Y. (2014). A review of non-contact micro- and nano-printing technologies, Journal of Micromechanics and Microengineering, Vol. 24, No. 5, Article 053001, doi: 10.1088/0960-1317/24/5/053001.

[3] Šafarič, R., Lukman, D. (2014). One-finger gripper based on the variable van der Waals force used for a single nano/micro-sized object, Journal of Micromechanics and Microengineering, Vol. 24, 1-13, doi: 10.1088/0960$1317 / 24 / 8 / 085012$.

[4] Uran, S., Šafarič, R., Bratina, B. (2017). Reliable and accurate release of micro-sized objects with a gripper that uses the capillary-force method, Micromachines, Vol. 8, No. 6, 182, doi: 10.3390/mi8060182.

[5] Kudryavtsev, Y.V., Gelinck, E., Fischer, H.R. (2009). Theoretical investigation of van der Waals forces between solid surfaces at nanoscales. Surface Science, Vol. 603, No. 16, 2580-2587, doi:10.1016/i.susc.2009.06.007.

[6] Rowland, R.S., Taylor, R. (1996). Intermolecular nonbonded contact distances in organic crystal structures: Comparison with distances expected from van der Waals radii, The Journal of Physical Chemistry, Vol. 100, No. 18, 7384-7391, doi: 10.1021/ip953141.

[7] Alvarez, S. (2013). A cartography of the van der Waals territories, Dalton Transactions, Vol. 42, 8617-8636, doi: $10.1039 / \mathrm{c} 3 \mathrm{dt} 50599 \mathrm{e}$.

[8] Rumpf, H. (1990). Particle Technology, Chapman and Hall, London, UK.

[9] Rabinovich, Y.I., Adler, J.J., Ata, A., Singh, R.K., Moudgil, B.M. (2000). Adhesion between nanoscale rough surfaces I. Role of asperity geometry, Journal of Colloid and Interface Science, Vol. 232, No. 1, 10-16, doi:10.1006/jcis. $\underline{2000.7167 .}$ 
[10] Rabinovich, Y.I., Adler, J.J., Ata, A., Singh, R.K., Moudgil, B.M. (2000). Adhesion between nanoscale rough surfaces II. Measurement and comparison with theory, Journal of Colloid and Interface Science, Vol. 232, No. 1, 17-24, doi:10.1006/jcis.2000.7168.

[11] Katainen, J., Paajanen, M., Ahtola, E., Pore, V., Lahtinen, J. (2006). Adhesion as an interplay between particle size and surface roughness, Journal of Colloid and Interface Science, Vol. 304, No. 2, 524-529, doi: 10.1016/i.jcis.2006. $\underline{09.015}$.

[12] Matope, S., van der Merwe, A.F., Nemutudi, R., Nkosi, M., Maaza, M. (2011). Micro-material handling employing Ebeam generated topographies of copper and aluminium, The South African Journal of Industrial Engineering, Vol. 22, No. 2, 175-188, doi:10.7166/22-2-24.

[13] Matope, S., van der Merwe, A.F., Rabinovich, Y.I. (2013). Silver, copper and aluminium coatings for micromaterial handling operations, The South African Journal of Industrial Engineering, Vol. 24, No. 2, 69-77, doi: 10.7166/24-2-554.

[14] Eichenlaub, S., Gelb, A., Beaudoin, S. (2004). Roughness models for particle adhesion, Journal of Colloid and Interface Science, Vol. 280, No. 2, 289-298, doi:10.1016/i.jcis.2004.08.017.

[15] Škorc, G., Šafarič, R. (2012). Adaptive positioning of MEMS production system with nano-resolution, Intelligent Automation \& Soft Computing, Vol. 18, No. 4, 381-398, doi: 10.1080/10798587.2012.10643250.

[16] Lambert, P. (2007). Capillary Forces in Microassembly, Springer, Boston, USA.

[17] Kraut, B. (2003). Strojniški priročnik, Založba Litostroj, (in Slovene), Ljubljana, Slovenia.

[18] Parsegian, V.A. (2006). Van der Waals Forces, Cambridge University press, UK.

[19] Montgomery, S.W., Franchek, M.A., Goldschmidt, V.W. (2000). Analytical dispersion force calculation for nontraditional geometries, Journal of Colloid and Interface Science, Vol. 227, No. 2, 567-584, doi: 10.1006/jcis.2000. $\underline{6919}$.

[20] Visser, J. (1976). Adhesion of colloidal panicles, In: Matijevic, E., Borkovec, M. (eds.), Surface and Colloid Science, Vol. 8, John Wiley \& Sons, New York, USA, 3-84.

[21] Kirsch, V.A. (2003). Calculation of the van der Waals force between a spherical particle and an infinitive cylinder, Advances in Colloid and Interface Science, Vol. 104, No. 1-3, 311-324, doi: 10.1016/S0001-8686(03)00053-8.

[22] Šafarič, R., Rojko, A. (2007). Inteligentne regulacijske tehnike v mehatroniki, (in Slovene), Tiskarna tehniških fakultet, Maribor, Slovenia.

[23] Lipkin, D.M., Israelachvili, J.N., Clarke, D.R. (1997). Estimating the metal-ceramic van der Waals adhesion energy, Philosophical Magazine: A, Vol. 76, No. 4, 715-728, doi: 10.1080/01418619708214205.

[24] Ahmadi, G. London-van der Waals Force, Clarkson University, from https://webspace.clarkson.edu/projects/crcd/ public_html/me437/downloads/5_vanderWaals.pdf, accessed January 25, 2018.

[25] Attraction - Dispersion energies (2008), from http://soft-matter.seas.harvard.edu/index.php/Attraction - Dispersion_energies, accessed January 10, 2018.

[26] Bondi, A. (1966). Van der Waals volumes and radii of metals in covalent compounds, The Journal of Physical Chemistry, Vol. 70, No. 9, 3006-3007, doi: 10.1021/j100881a503.

[27] Batsanov, S.S. (2001). Van der Waals radii of elements, Inorganic Materials, Vol. 37, No. 9, 871-885, doi: 10.1023/ A:1011625728803. 


\section{APEM}

\title{
Določanje nanohrapavosti mikropredmetov z merjenjem van der Waalsove sile
}

\author{
Bratina, B..$^{a,}{ }^{*}$, Šafarič, J. ${ }^{a}$, Uran, S. ${ }^{a}$, Šafarič, R. ${ }^{a}$ \\ ${ }^{a}$ Faculty of Electrical Engineering and Computer Science, University of Maribor, Maribor, Slovenia
}

\section{POVZETEK}

Tehnike 3D tiskanja ali montaže v mikro/nano svetu omogočajo izdelavo mikropredmetov za gradnjo majhnih strojev ali struktur za potrebe biomedicine, kot je gojenje živih celic na področju tkivnega inženiringa. Sestavljanje na nivoju mikrovelikosti zahteva avtomatizirane postopke manipulacije in metode za določanje primernih predmetov za sestavljanje. Slednje je mogoče z meritvijo Van der Waalsove sile in določitvijo razdalje med dvema kontaktnima objektoma pri največji Van der Waalsovi sili. Odvisni niso le od Hamakerjevih koeficientov materialov $\mathrm{v}$ stiku in njihove geometrije, temveč tudi od nanohrapavosti in kristalne strukture kontaktnih površin. Predstavljena je metoda za merjenje Van der Waalsove sile in določanje razdalj med mikropredmeti (velikosti 10-100 $\mu \mathrm{m}$ ) med materiali, ki so v stiku pri največji Van der Waalsovi sili, ob prisotnosti nanohrapavosti in hrapavosti kristalne strukture. Predlagani model je bil potrjen z eksperimentalnimi laboratorijskimi rezultati na različnih materialih in oblikah (steklene in polistirenske kroglice, kovinske žice).

\section{PODATKI O ČLANKU}

Ključne besede:

Mikropredmet;

Hrapavost površine;

Nanohrapavost;

Van der Waals sila;

Razdalja pri največji van der Waals sili

*Kontaktna oseba: bozidar.bratina@um.si

(Bratina, B.)

Zgodovina članka:

Prejet 14. septembra 2018

Popravljen 18. februarja 2019

Sprejet 24. februarja 2019 